\section{Toward Quantitative Defect Analysis Using HREM}

David J. Smith, Arizona State University

The electron microscope has evolved to the level where it is now straightforward to record high-resolution images from thin samples ( $t \sim 10$ to $20 \mathrm{~nm}$ ) that are directly interpretable in terms of atomic arrangements. Whilst recorded images necessarily represent two-dimensional projections of the structure, many defects such as dislocations and interfaces may be linear or planar in nature and this might be expected to be amenable to detailed characterization. In this review, we briefly consider the recent significant progress that has been made in quantitative defect analysis using the high-resolution electron microscope and then discuss some drawbacks to the technique as well as potential scope for further improvements. Surveys of defect modeling for some small-unit-cell materials ${ }^{1}$ and interfaces ${ }^{2}$ have been recently published, and reference should be made to other papers in this symposium for further examples.

The technique of structure imaging originated in the early 70 s with observations of large-unit-cell block oxides ${ }^{3,4}$. Image interpretation was initially validated by prior knowledge of the crystal structure, whereas it became commonplace in later studies to verify postulated structural models on the basis of agreement between experimental micrographs and calculated images, mostly simulated using the multislice algorithm ${ }^{5}$. The comparisons were usually qualitative and somewhat subjective, but the models were generally considered as more acceptable if an image "match" was achieved for more than one micrograph from a through-focal series ${ }^{6}$. It has, however, been established that the apparent locations of atomic columns at aperiodic features such as interfaces may vary by as much as $0.03 \mathrm{~nm}$ depending on the defocus value ${ }^{7}$. If structure refinement to this level of accuracy or better is desired, then improved methods for establishing the actual defocus values used for image recording must therefore be developed. In this regard, the use of automatic cross-correlation tech-

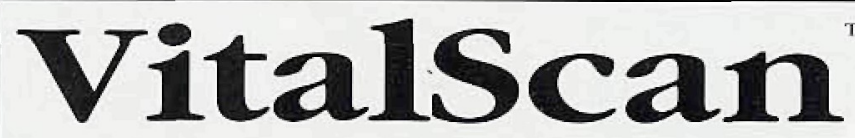

\section{Modernize your SEM with Digital Imaging Technology}

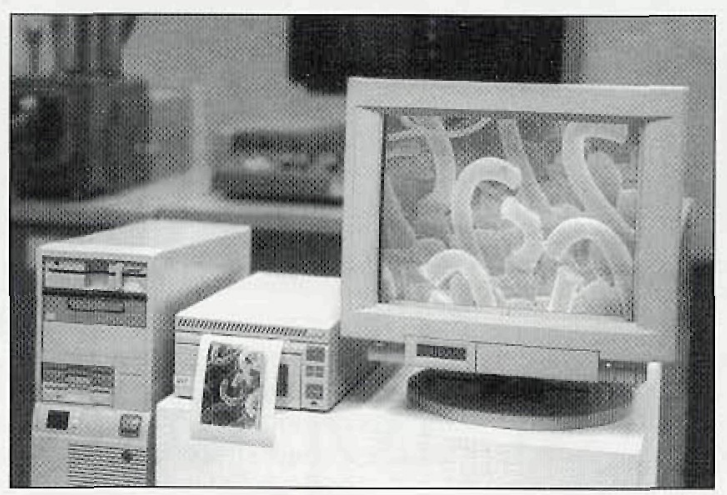

- ACTIVE BEAM CONTROL

- UP TO 4006×4096 CAPTURE (12-BIT Digitization)

- DUAL ADCS (Collect Secondary and Backscatter)

- FRAME and PIXEL AVERAGING
- AUTOMATIC CONTROL CONTRAST/BRIGHTNESS

- OPTIONAL FAST X-RAY MAPPING

- OPTIONAL IMAGE ANALYSIS SOFTWARE

\section{Vital Image Technology • 800-860-IMAGE}

Vital Image Technology offers photographic-quality printers and other imaging related products such as CCD cameras, frame grabbers and scanners. VIT has the imaging solution for both network and stand alone applications. niques to compare the Fourier coefficients of diffracted beams in reciprocal space appears to be rapid, reliable and thus highly practical for evaluating periodic image features in the vicinity of the defect of interest ${ }^{3}$. Some studies have been published wherein the residual differences between experimental and calculated images have been minimized significantly using least-squared refinement methods, despite the need for correction of photographic nonlinearities (see, for example, Ref. 9). Neverless, such successes are by no means universal - as exemplified in recent work on $\mathrm{Ga}_{x} \mathrm{Al}_{1 . x}$. As where the serious mismatch between image intensity, contrast and pattern appearance defied an exhaustive search to establish a conclusive cause for the differences ${ }^{10}$.

There are several factors that clearly could markedly affect the reliability of the refinement process, especially in achieving quantitative agreement between experimental and simulated micrographs. For example, image simulation programs utilize atomic scattering factors that may not be sufficiently accurate and, because of limited sampling in reciprocal space, approximations are invariably made for electron scattering in the vicinity of defects. On the practical side, multiple and inelastic scattering, amorphous surface overlayers, and electron irradiation damage may all influence the integrity of the recorded image to a greater or lesser (unknown?) extent. For example, structural modification, especially for atoms in the vicinity of lattice defects and surfaces, is inevitable in the face of the high beam current densities required for image recording at very high magnification. Total beam exposure should therefore be restricted whenever possible. Surface oxide or contamination layers, oftentimes caused by sample preparation or even exposure to air, must degrade the appearance of the final image which originates from electron transmission through the entire projected sample. In the absence of signal averaging, which is obviously inapplicable to aperiodic defects, image quantification can be substantially improved through careful attention to minimizing surface generated noise Inelastic scattering cannot be avoided nor can its effects be easily incorporated into image simulations because of the lack of reliable information about the energy (and angular) spread associated with scattering of electrons from

Continued on Page 10

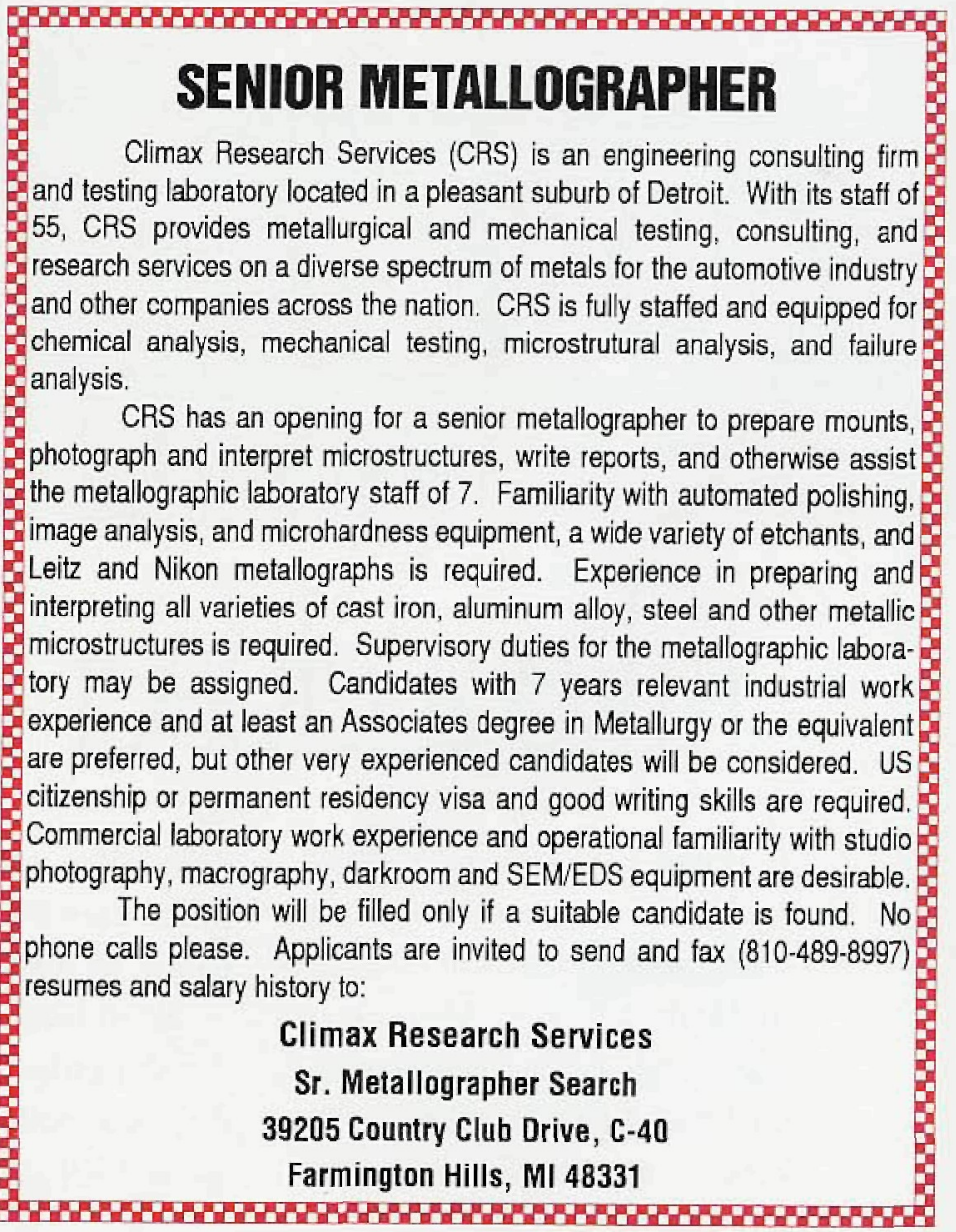




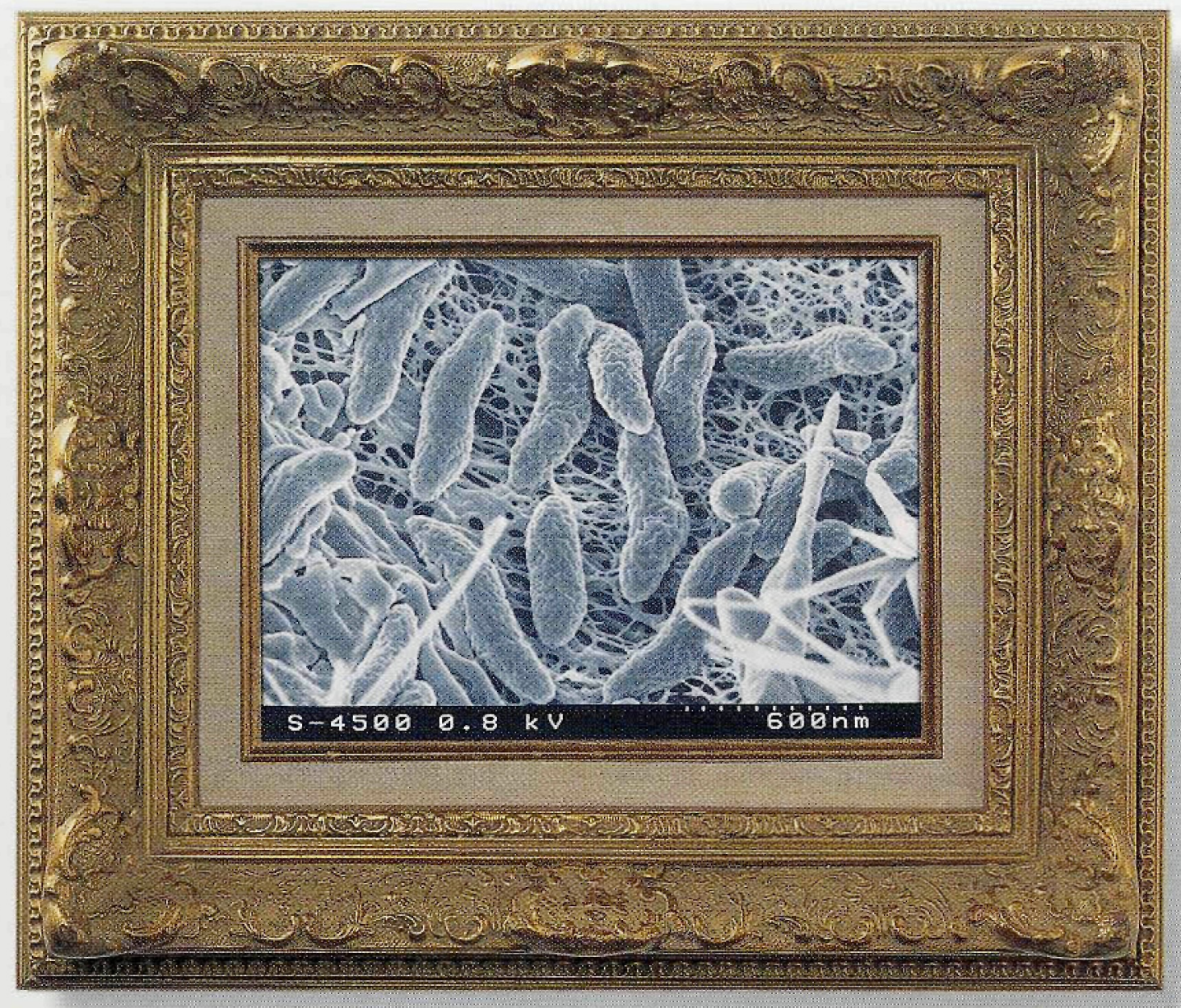

\section{This is the original. This is what you get with our passive capture imaging system.}

Sad, but true. Ordinary digital-image-capture systems, relying on digital beam control, just can't preserve the image quality offered by your microscope. It's your loss. And a big one, considering all you could do with a high-quality digitized image.

Our passive capture imaging ( $\mathrm{PCI}$ ) system, on the other hand, gives you images identical to the originals. Because it detects and uses the microscope's own scan signals, actually duplicating the scan generator's resolution up to 4000 lines - while microscope operation continues as usual.

Next, our easily used, Windowsbased PCI lets you do just about any-

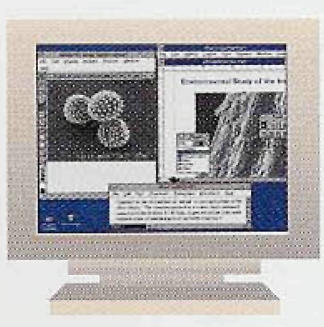

thing imaginable with that superior image. Annotate it. Store it as a TIFF file. Archive and combine it with data from other microscopes. Measure it. Resize it. Color it - with up to 16 million colors. Print it out. Place it in documents. Send it across the lab or over the Internet. And (try to find this elsewhere) even replay it to your microscope's photo CRT.

Oh, yes. PCI works with any SEM or STEM. Same with TEM and optical microscopes, where PCI uses a slow-scan CCD camera to acquire sharp, non-interlaced, square-pixel $1024 \times 768$ images.

Call now for a $\mathrm{PCl}$ demonstration. Because, if you'd like to do any of 1001 things with your microscope's image, you'll want to capture it perfectly. Without letting its quality get away.

\section{HITACHI}

SCIENTIFIC INSTRUMENTS

Nissei Sangyo America, Ltd.

755 Ravendale Drive

Mountain View, CA 94043 800-227-8877

E-mail: sidsales@nissei.com wwwnissei.com

25 West Watkins Mill Road Gaithersburg, MD 20878 800-638-4087

All trademarks are the property of their respective companies 
defects. The use of energy imaging filters could, however, facilitate image recording with only those electrons that have not lost energy in traversing the specimen - loss of contrast from the out-of-focus energy-loss electrons could thereby be alleviated or possibly removed ${ }^{12}$. In some special cases, where the defect of interest is close to the edge of the sample, it might instead be possible to utilize the off-axis electron hologram which is energy-filtered ${ }^{13}$. Finally, whilst multiple scattering is difficult to incorporate reliably into dynamic calculations, its effect can at least be minimized by restricting imaging to very thin regions.

Several possibilities are available for improving the speed, reliability and/or accuracy of the defect analysis. It is obvious, for example, that digital recording with a slow-scan CCD camera provides a high DQE with wide dynamic range, and hence better counting statistics (signal-to-noise ratio) whilst also avoiding the nonlinearities associated with photographic recording ${ }^{14}$. However, for some applications the effective pixel size referred to the sample needs to be of the order of $0.01 \mathrm{~nm}$ or smaller but then the typical $1024 \times 1024$ pixel field of view could be considered as being restrictive (although interpolation techniques to locate the exact positions of contrast maxima and minima could be used to alleviate this limitation). None of the defect analyses so far published appear to have been carried out under computer-controlled operation of the microscope. Nevertheless, it is abundantly clear that location of the coma-free axis for beam alignment, adjustment of objective lens astigmatism and selection of defocus can be routinely accomplished using computer control with greater accuracy than that attainable by even the experienced microscope operator $^{15}$. Subsequent defect refinement should thus be significantly simplified since it would not be necessary to take extraneous factors into account ${ }^{10}$. Improved image resolution is not likely to have a great impact on defect analysis at this stage of development since it is really signal-to-noise rather than resolution per se that determines the precision with which atomic columns can be located. However, the extra flexibility of imaging in additional projections should certainly facilitate the development of more accurate three-dimensional defect models. Finally, it appears that more attention needs to be given to the structure refinement process itself, in particular the problems of optimizing the recursive algorithm (what parametersiwhat accuracy?) and minimizing the residual discrepancy between experimental and simulated images ${ }^{16}$. The possible influence on the refinement of factors unrelated either to the structure or the model has been pointed out ${ }^{16}$, and some of the issues relating to establishing uniqueness or "goodness of fit" have also been discussed

In conclusion, defect analysis with the high-resolution electron microscope has now reached the stage where truly quantitative comparisons with stuctural models has become a reality. Applications to an increasing variety of materials over the next several years can be safely predicted and evaluation of different theoretical approaches to modeling might even be feasible ${ }^{17}$.

1. D.J. Smith, W.J. de Ruijter, M.R. McCartney and J.K. Weiss, Ultramicroscopy 52 (1993) 591

2. U. Dahmen, MSA Bulletin, June 1994, to be published

3. S. lijima, J. Appl. Phys. 42(1971) 5891 .

4. J.L. Hutchison and J.S. Anderson, phys. stat sol. (a)9(1972) 207

5. P.A. Goodman and A. Moodie, Acta Cryst. A30 (1974) 280.

6. J.C. Barry, Phil. Mag. A46 (1991) 111.

7. W. S. Saxton and D. J. Smith, Ultramicroscopy 18 (1985) 39

8. A. Thust and K. Urban, Ultramicroscopy 45 (1992) 23

9. W. King and B.S. Lamver, in Microbean Analysis 1991, p. 217

10. M. Hytch and W.M. Stobbs, Ultramicroscopy 53 (1994) 191.

11. J.M. Gibson and M. L. McDonald, MRS Symp. Proc. 82 (1987) 109

12. W.M. Stobbs and W O. Saxton, J. Microscopy 151 (1988) 171

13. M.R. McCartney and M. Gajdardziska-Josifovska, Ultramicroscopy 53 (1994) 283

14. W.J. de Ruijter and J.K. Weiss, Rev, Sci. Instr, 63 (1992) 4314

15. W.O. Saxton, D.J. Smith and S.J. Erasmus, J. Microscopy 130 (1983) 187

16. D. Hofmann and F. Ernst, Ultramicroscpy 53 (1994) 205.

17. The Center for High Resolution Electron Microscopy at Arizona State University is supported by NSF Grant DMR-9115680

Reprinted from Proceedings, 1994 Microscopy Society of America Conference.

KEVEX FLAME $^{\mathrm{m}}$ Fuzzy Logic Automated Microanalysis Engine.... The NEW Macintosh Based EDS Microanalyzer from Kevex Instruments!

Kevex FLAME is a Macintosh based EDS analyzer, affordably priced, offering a flexible architecture and user-friendly GUI. FLAME is available as a retrofit (i.e. upgrading older EDS analyzers) or as a new and complete standalone microanalyzer, which includes:

\section{- Fuzzy Logic \& Artificial Intelligence database}

- User-definable setups with custom preferences

\section{- Image and X-ray map acquisitions up to 4096 pixels}

\section{- Feature Analysis with comprehensive parameters and Image "Gallery"}
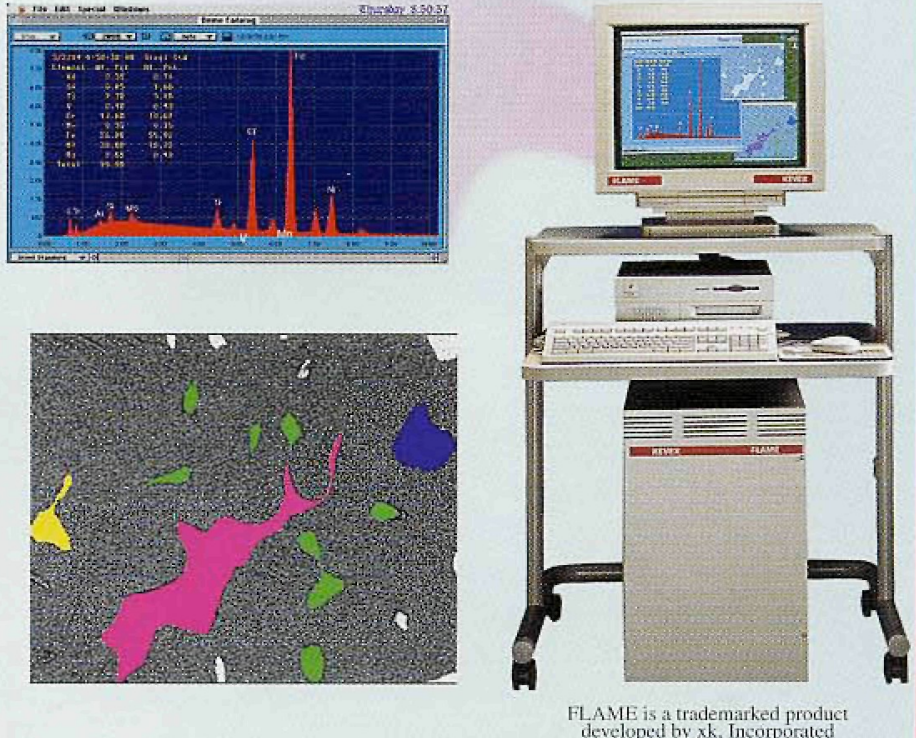

Kevex Instruments • 24911 Avenue Stanford • Valencia, CA 91355 • Tel. (805) 295-0019 • Fax (805) 295-8714 


\section{GOT AN AFM YET?}

Need more resolution than your optical microscope can supply? Tired of the tedious, time consuming, and often damaging sample prep for electron microscopy (EM) and other techniques? Intrigued by AFM force measurements?

See What You've Been Missing-the BioScope AFM

The BioScope ${ }^{\text {TM }}$ Atomic Force Microscope (AFM) mounts on popular inverted microscopes and pro-

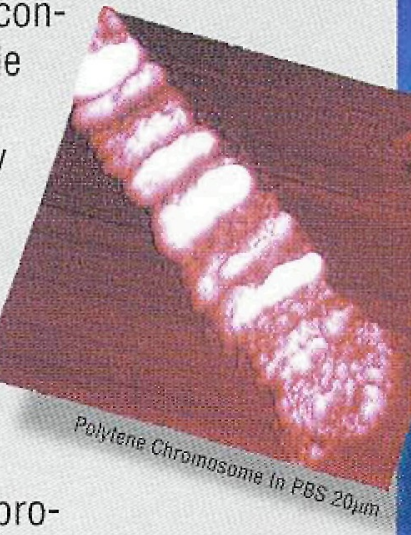
vides 200 times the resolution of optical microscopy. Use the optical microscope to locate areas of interest, then scan with true 3-D imaging with resolution comparable to or better than EM. Image your specimens directly in petri dishes, on glass slides or coverslips, in air or biological fluids-no vacuum drying, rigid design and propri-

etary TappingMode ${ }^{\mathrm{TM}}$ technology ensure that you get superior images without sample damage. The BioScope AFM-designed specifically for the life scientist by the world leader in AFM. Call for a free application note and brochure.

See us at MSA, Booth 714

\section{Digital
Instruments}

Santa Barbara, California, 800-873-9750, 805-899-3380 